\title{
Literaturunterricht für tschechische DaF-Studierende: Vorüberlegungen im Ausblick auf ein curriculares Kolloquium
}

\author{
Jan Budňák
}

The PISA survey discovered grave deficiencies in the reading skills of Czech pupils. The paper gives some thoughts and suggestions on how literature classes at departments of foreign language of Faculties of Education could support the development of the students' reading skills. Thus, the prerequisites for their own manipulation with literature in their future teaching practice might be given.

reading skills - teaching literature - methodology of teaching literature - foreign language teaching

Lesekompetenz - Literaturunterricht - Literaturdidaktik - DaF-Unterricht

Das deutsche PISA-Konsortium definiert die Lesekompetenz als die Fähigkeit, „geschriebene Texte zu verstehen, zu nutzen und über sie zu reflektieren, um eigene Ziele zu erreichen, das eigene Wissen und Potenzial weiterzuentwickeln und am gesellschaftlichen Leben teilzunehmen." ${ }^{\prime}$ Auf den ersten Blick ist die Weitläufigkeit der Definition sichtbar - allerdings auch ihre Stringenz. Leseverstehen wird hier als ein Netzwerk aus Aufmerksamkeit, Erfahrung, Können und Wissen dargestellt und im internationalen Vergleich auch überprüft. Einzelne Dimensionen, die die Leseaufgaben der PISA-Studie bei etwa fünfzehnjährigen Schülern zu erfassen trachten, sind: a) Informationen aus einem Text zu extrahieren, b) ein allgemeines Verständnis des Textes zu entwickeln, c) eine textbasierte Interpretation zu entwickeln, c) über den Inhalt des Textes zu reflektieren, und schließlich d) über die Form des Textes zu reflektieren. ${ }^{2}$ Dabei kommt den sog. „fortlaufenden“'Texten, deren Großteil belletristische Texte ausmachen, eine zentrale Stellung zu: Es werden nicht nur inhaltslose, abzählreimartige Gedichtchen oder Stichwortkataloge herangezogen, wie von Kritikern manchmal spöttisch angemerkt wird. Gerade bei den „fortlaufenden“ Textsorten kommt es jedoch auf die verhältnismäßig fortgeschrittenen Komponenten der Kompetenz Leseverstehen an: insbesondere auf die Leserorientierung aufgrund verschiedener formaler (z. B. Deixis, Erzählsituation) und inhaltlicher (z. B. Situation) Textgliederungssignale. Obwohl z. B. ein fünfzehnjähriger Leser diese Begriffe natürlich nicht zu lernen braucht, muss er dennoch, und zwar mit nicht zu unterschätzendem Aufwand lernen, wie man sich danach im Text orientiert und dadurch - um mit PISA zu formulieren - „eine textbasierte Interpretation

1 http://pisa.ipn.uni-kiel.de/index.html, aufgerufen am 24. April 2009

2 vgl. ebd. 
entwickelt“. Im Jahr 2009, nach drei durchgeführten PISA-Untersuchungen (2000, 2003, 2006), ist wohl unnötig zu ergänzen, dass tschechische Schüler bei der Untersuchung der Lesekompetenz im Durchschnitt recht miserabel abschneiden. Die Homepage des tschechischen Bildungsministeriums stellt die diesbezüglichen PISA-Ergebnisse von 2007 wie folgt vor: „Oproti výsledkům v přírodních vědách a v matematice jsou výsledky českých žáků v oblasti čtení podprůměrné; toto zjištění se nijak neliší od výsledků předchozích dvou šetření v letech 2000 a 2003. Statisticky významně lepší výsledky měli žáci 21 zemí. “1

Herausforderungen, vor die diese durch die PISA-Studien offenbarte Lage die fremdsprachlichen Institute der Pädagogischen Fakultäten, und insbesondere deren Literaturunterricht stellt, sind im Grunde von zweierlei Art. Zum einen handelt es sich um eine fachdidaktische Herausforderung. Hier gilt es, Studierende (also angehende LehrerInnen) für eine textbasierte und kontextualisierende Beschäftigung mit „fortlaufenden“ Texten entsprechend auszurüsten. Dabei können sicherlich auch fachdidaktische Fächer Wesentliches beitragen, obwohl die Annahme, dass zukünftigen Lehrkräfte in Ihrem Unterricht später nur deshalb mit Literatur arbeiten werden, weil sie in diesem Bereich über methodische Kenntnissen verfügen, eher Misstrauen weckt. Zum anderen darf aber nicht übersehen werden, dass die Studierenden selbst „Produkte“ des tschechischen Schulsystems sind, das besonders im Hinblick auf die Entwicklung der Lesekompetenz „urlaubsreif“ ist. Die angehenden LehrerInnen müssen sich also zuallererst zumindest die Grundsätze der Lesekompetenz aneignen, was nicht ohne ausgedehnte Beschäftigung mit entsprechend anspruchsvollen „fortlaufenden“ bzw. belletristischen Texten denkbar ist. Sie müssen einfach zunächst ein Training absolvieren, wie gelesen wird, bevor sie lernen, wie Leseverstehen vermittelt wird.

Leider ist der gegenwärtige Organisationsrahmen des Literaturunterrichts etwa an den Instituten für deutsche Sprache vieler nicht-philologischer Studienprogramme in Tschechien diesbezüglich nicht eben erfreulich. Das, was bei Diplomprüfungen gefordert wird und worauf die Studierenden folglich vorbereitet werden müssen bzw. vorbereitet werden wollen, ist mehr oder weniger Literaturgeschichte. Darüber hinaus können auch kaum schwerpunktmäßig bzw. thematisch orientierte Kurse ${ }^{2}$ angeboten werden, da die für Literatur eingeräumte Zahl an Kreditpunkten mit

1 „Im Vergleich zu den Ergebnissen in naturwissenschaftlichen Fächern und in Mathematik sind die Ergebnisse tschechischer Schüler im Bereich Leseverstehen unterdurchschnittlich; diese Feststellung unterscheidet sich in keiner Weise von den Ergebnissen der vorigen zwei Untersuchungen von 2000 und 2003. Statistisch erheblich bessere Ergebnisse haben Schüler aus 21 anderen Ländern erzielt." (http://www.msmt.cz/pro-novinare/vysledky-mezinarodniho-vyzkumu-pisa-2006, Übersetzung J. B.)

2 Solche Kurse - z. B. „Kurzprosa des Expressionismus“, „Wien um die Jahrhundertwende“, „Dorfgeschichte“, „Bürgerliches Trauerspiel“ oder meinetwegen „Flaneure in der deutschsprachigen Literatur" - haben ein viel höheres Potential im Hinblick auf analytisches Lesen als etwa „Deutsche Literatur II: Barock bis Vormärz", da sie auf zahlreichen Ebenen zum Vergleichen der Texte animieren. Dabei hängt es nur von der Lehrkraft ab, dass die Diskussionen nicht ins Abstrakt-Akademische abgleiten, sondern die ethische oder geistesgeschichtliche Dimension des Textes (textnah) erörtern.

Brünner Hefte zu Deutsch als Fremdsprache • Jahrgang 2 •Nummer $1 \bullet 2009$ 
Müh und Not die nach Epochen geordneten Pflichtkurse abdeckt. (In ähnlicher Lage befinden sich auch die linguistischen Disziplinen). Die Prüfungsansprüche kommen überdies (noch immer!) der Erwartung bzw. Vorerfahrung vieler Studierender in punkto „Literaturunterricht“ - er bestehe aus Listen von biographischen Daten und komprimierten Inhaltsangaben - sehr gelegen. Diesem ausgesprochen schädlichen und der Forderung nach Entwicklung der Lesekompetenz geradezu zuwider laufenden Automatismus will ich im folgenden Text einige alternative Vorschläge entgegensetzten. Gerade an Pädagogischen Fakultäten muss die oft ohne Hinterfragen angenommene Gleichung „Literatur" ist gleich „Literaturgeschichte“ durch die folgende ersetzt werden: „Literatur“ ist gleich „Lesekompetenz“ im Sinne der anfangs angeführten Definition inklusive - auf höchster Ebene - der epochenspezifischen (geistesgeschichtlichen) Kontextualisierung. ${ }^{1}$

\section{Reader: Verbindliche - und verbindende - Lektüre von kürzeren Prosatexten}

Der erste Vorschlag, wie die Entwicklung der Lesekompetenz anhand von literarischen Texten anzubahnen wäre, besteht in der einfachen Überlegung, dass kurze Texte überschaubarer sind, im Tempo ein Stück pro Woche bezwungen werden können und - vor allem - sich „kooperativ“ aufarbeiten lassen. Diesen Punkt halte ich für den wichtigsten: Alle Kursteilnehmer haben den Text gelesen ${ }^{2}$ (wenn auch mit oberflächlichem Verständnis), und nun sollen sie üben können, ihre Hypothesen diesbezüglich zu formulieren, die konträren Hypothesen zu überprüfen auf bestimmte Punkte aufmerksam zu machen. All dies mit dem Text als der alleinigen Fundgrube für ihre Argumente.

Soll dann der durch die Diplomprüfung gestellte Imperativ der „EpochenKenntnisse" saturiert werden, sehe ich einen einzigen Ausweg: eine "Anthologie“ von kurzen Texten, die möglichst deutlich die wesentlichen Zeichen der Epoche tragen. ${ }^{3}$ Für eine solche Sammlung von Texten hat sich an deutschen Universitäten der englische Begriff „Reader" eingebürgert. Neben seiner „Kompaktheit" besteht der größte Vorteil eines solchen Readers darin, dass er eine Basis für die gemeinsame (geregelte) Analyse des Textes bildet. Haben alle Teilnehmer einen überschaubaren

1 In der Definition der Lesekompetenz wird übrigens der „Missbrauch“ literarischer Texte zu ausschließlich grammatikalischen Unterrichtszielen nicht erwähnt. Auch ich werde diesen fragwürdigen Ansatz im Weiteren nicht berücksichtigen, obwohl diese veraltete Methode aufgrund ihrer Zählebigkeit explizite Ächtung verdienen würde.

2 In diesem Zusammenhang kann eine autoritative Maßnahme nicht unerwähnt bleiben. Die Motivation, die Pflichtlektüre zu lesen, muss natürlich auch dadurch gestärkt werden, dass am Semesterende wirklich das Verständnis des Gelesenen abgeprüft wird, nicht etwa Figurennamen oder Inhaltsangaben, die zweifelsfrei mit weniger „Aufwand“ der Wikipedia zu entnehmen wären.

3 Eine alternative Lösung ist natürlich, etwa einen Roman pro Semester gründlich zu untersuchen, um die sonst notwendige Gesamtschau aus der Vogelperspektive am jeweiligen Beispiel zu konkretisieren. Andererseits lassen sich aus einer einzigen Erzählung, und sei sie auch von Schiller, nicht die Merkmale von den Epochen Barock bis Vormärz herleiten. 
und halbwegs aufbereiteten ${ }^{1}$ Text im Wortlaut vor sich, können alle zur Diskussion etwas beitragen.

\section{Biographien, Inhaltsangaben, Eindrücke}

Um der oben erwähnten Vorerwartung, die Literatur auf (oft oberflächliche) Fakten aus der Literaturgeschichte reduziert, nicht Vorschub zu leisten, sollten in dem auf Lesekompetenz ausgerichteten Literaturunterricht zunächst einige der „traditionellen“ Bestandteile auf ihre Zweckmäßigkeit hin überprüft werden. Wodurch wird in Literaturstunden am häufigsten die Auseinandersetzung mit dem Text vereitelt? Ohne unmäßige Übertreibung lässt sich sagen, dass diese Elemente, die sich mit der landläufigen Vorstellung von „Literaturunterricht“ oft zu hundert Prozent decken: (Ausufernde) Lebensläufe der Autoren und (ermüdende) Inhaltsangaben, die die wichtigen Momente aus den Augen verlieren. Diese Elemente des Literaturunterrichts breiten sich aber oft auf Kosten der Textarbeit übermäßig aus. Verzichtet man auf $\operatorname{sie}^{2}$ bzw. will man ohne sie auskommen, stellt sich auf Seiten der Kursteilnehmer zunächst eine allgemeine Ratlosigkeit ein: Was sei Literatur denn sonst noch, wenn nicht Lebensläufe, Daten- und Namenslisten oder Inhaltsangaben? Das oft von ungespielter Bestürzung begleitete Aha-Erlebnis muss seitens der Lehrenden richtig aufgefangen werden, denn es droht in ein anderes Stereotyp umzuschlagen, das ebenso beständig im Hinblick auf schöne Literatur tradiert wird. Es ist die Vorstellung ${ }^{3}$, dass literarische Texte keine „objektive“ Aussage haben, dass sie eben zu abgehoben und undurchsichtig sind und dazu von im Moment nicht ansprechbaren Individuen verfasst wurden. Folglich lohne es sich nicht, sich damit näher zu beschäftigen, die Texte könnten höchstens Eindrücke hervorrufen, die aber völlig subjektiv seien. Die entsprechende Erörterungsform eines so begriffenen literarischen Textes könne dann nur der Erfahrungsbericht sein. Was bei dieser Annahme („Literatur ist subjektiv“) ebenso zu kurz kommt wie bei der ersten („Literatur ist gleich Daten“), ist der Text. ${ }^{4}$

1 Als Fokussierung der Aufmerksamkeit auf wichtige „Warum“-Fragen bei der Lektüre zuhause empfehlen sich natürlich Möglichkeiten des E-Learning. Dies sind an erster Stelle direkte Fragen zum Textverständnis, weiter auch die auf unterschiedliche Fragestellungen ausgerichteten „Diskussionsfaden“, Recherchetipps (vor allem bei jüngerer Literatur) usf.

2 Die Rede ist hier von Seminaren zu verschiedenen literarischen Epochen. Für Vorlesungen gilt dies freilich nicht: Gerade sie sind der Ort, wo diese Informationen - durch Kontextualisierung verständlich gemacht - zur Sprache kommen.

3 In diesem Zusammenhang muss auf die jüngere Geschichte unserer Lande hingewiesen werden, die das halbe Jahrhundert Diktatur (1939-1989) wesentlich beeinflusst hat. Werden von allerlei Instanzen allerlei Texte missbraucht, verdreht, als Verfolgungsinstrumente benutzt oder ganz entleert, kann sich keine breit akzeptierte und gepflegte „hermeneutische“ Kultur entwickeln.

4 Für jemanden, der auch nur gewisse Grundkenntnis von Literatur(-wissenschaft) hat, sind diese Überlegungen sicher so selbstverständlich wie Sonnenauf- und -untergang. Ich hätte vor fünf Jahren auch nicht geglaubt, wofür Literatur - selbst von (Fremdsprachen-)Lehrenden und Studenten von geisteswissenschaftlichen Disziplinen - gehalten werden kann. 


\section{3. "Regie" der Stunde, Diskussionsleitung}

Nun wird also in einer literaturwissenschaftlichen Lehrveranstaltung seminarartiger Form an einem Institut für Deutsch einer Pädagogischen Fakultät ein „Embargo“ über biographische Angaben zum Autor, auf unreflektierte Wiedergabe des Inhalts und auf nicht verifizierbare „Lektüreeindrücke“ verhängt. Das Ziel leuchtet ein: das Textverständnis zu fördern, die „Lesekompetenz“ zu üben. Wie soll der allgemeinen Panik, die die häufigste Folge von diesen Einschränkungen ist, begegnet werden? Die Lösung, die am sichersten das Textverständnis gewährleistet, wäre die „Regie“ der Stunde ganz der Lehrkraft vorzubehalten. Ist der Kursleiter bzw. die Kursleiterin kreativ genug, kann er/sie, ohne zu dozieren, die Aufmerksamkeit der Studierenden auf die Momente des Textes lenken, die tatsächlich zentral sind. Geeignete Aufgaben zur Behandlung von literarischen Texten im Unterricht zum Trainieren der „Lesekompetenz“ werden im nächsten Abschnitt skizziert.

Meine Erfahrung spricht allerdings dafür, dass die grundsätzlichen „Griffe“, die auch zu einer inhaltlichen Bearbeitung von Texten im Unterricht verwendet werden können, auch von den Studierenden als „Diskussionsleitern“ angewendet werden können, die den Text, zu dessen Verständnis sie ihren KommilitonInnen verhelfen sollen, nur partiell begriffen haben. Die Mängel können dann - mit Umsicht - von der Lehrkraft korrigiert werden. Der Ertrag der scheinbaren Zumutung, den Aufbau der Unterrichtseinheit und die Grundlinien des Interpretationsverfahrens von den (jeweils für einen Text zuständigen) Kursteilnehmern abhängig zu machen, ist potentiell sehr groß. Die Kursteilnehmer durchlaufen aufdiese Weise eine Einführung in die Literaturanalyse eigentlich jede Woche von neuem. Eine Bestandsaufnahme der Grundelemente des Textes (Figurenkonstellation, Zeitverhältnisse, Erzählsituation, Wendepunkte der Handlung, Motive, Symbole usf., je nach deren Wichtigkeit für den jeweiligen Text) eignet sich für den Einstieg in die Diskussion, es muss nicht lange nachgeblättert werden, kann - in variierender didaktischer Aufbereitung - das ganze Plenum beschäftigen und frischt die Erinnerung an den Text auf. Am Beginn der Stunde kann sie eigentlich auch von jemandem geleitet und didaktisch umgesetzt werden, der nur eine grobe Vorstellung hat, worauf sie hinausläuft.

\section{Aufbau der Einheit, Beispielaufgaben, Schreibaufgaben}

Es liegt nahe, eine Unterrichtseinheit zur Spätromantik etwa anhand von E. T. A. Hoffmanns Schauererzählung „Der Sandmann“ (1817) mit einer Erarbeitung der Figurenkonstellation zu eröffnen. Da das Geheimnisvolle der auf dem Doppelgängermotiv aufgebauten Geschichte gerade in der (eventuell nur in der Vorstellung des Protagonisten existenten) Identität von mehreren Charakteren besteht, wäre etwa die folgende Einstiegsaufgabe passend: „Welche Figuren kommen in der Erzählung vor? In welchen Situationen begegnen sie einander?“ Die 
dabei gesammelten Informationen können in Form eines „Beziehungsnetzes“ an der Tafel als Orientierungshilfe für weiterführende Aufgaben festgehalten werden. Für Einstiegsaufgaben gilt also Folgendes: Sie sollten alle Teilnehmer involvieren, ihre Lektüreerinnerungen aktivieren und selektiv Schwerpunkte setzen. Sie sind (offene) „Sammelfragen“, oft frontal gestellt und beantwortet. Meiner Erfahrung nach sind demgegenüber solche Fragen ungeeignet, die eine allgemeine Antwort verlangen, z. B. worum es im Text gehe (das führt meistens zur Nacherzählung von Handlungsabschnitten) oder wie der Text den Studierenden gefallen habe.

Üblicherweise wird der zweite Abschnitt der Stunde von detaillierterer Textarbeit eingenommen. Hiefür taugt m. E. die Gruppen- oder Paararbeit am besten, denn dadurch kann in kurzer Zeit viel Stoff bearbeitet - und präsentiert werden. Sprachliche Kompetenzen, die dabei gefördert werden, sind Leseverstehen, Schreiben (eine strukturierte, stichwortartige Antwort vorbereiten), Sprechen (dialogisch in Gruppe, monologisch bei der „Präsentation“) und Hörverstehen (die KommilitonInnen haben denselben Text gelesen, können also durchaus ergänzen bzw. richtig stellen). Natürlich hängt alles von der Fragenformulierung für die Gruppen ab; sehr empfehlenswert ist diesbezüglich eine Vorbesprechung der Lehrkraft mit den „Diskussionsleitern“ bzw. ein Hinweis auf potentielle Inspirationsquellen (vorformulierte Fragen im E-Learning, Sekundärliteratur usf.). Wichtig ist hier wieder die Verhinderung von Fragestellungen, die auf die Nacherzählung der Handlung („Was ist dann passiert?") ausgerichtet sind; „Warum“-Fragen, am besten mit Hinweisen auf entsprechende Textstellen bzw. Seitenzahlen, sind demgegenüber vorzuziehen. Bei Hoffmanns "Sandmann“ etwa bieten sich u. a. die folgenden Aufgabenstellungen an: „Das zentrale Leitmotiv der Geschichte sind Augen bzw. das Sehvermögen des Menschen und dessen Verzerrung. Suchen Sie im Text Beispiele für diese Verzerrungen der (optischen) Wahrnehmung und interpretieren Sie sie“ bzw. „Welche Ähnlichkeiten finden Sie zwischen den alchemistischen Versuchen, die Nathanaels Vater mit Coppelius (S. x) und Spalanzani mit Coppola (S. xy) machen? Was ist die logische Folge dieser Ähnlichkeiten?“ bzw. „E. T. A. Hoffmann wird als Repräsentant der sog. Schauerromantik gesehen. In welchen Szenen entsteht beim Leser der Eindruck einer bedrohlichen Unsicherheit? Wodurch wird er erzielt?" usf.

Meines Erachtens sind erst nach dieser eingehenden Textarbeit die textübergreifenden Fragen sinnvoll, die die Studierenden „über den Inhalt des Textes reflektieren" lassen, wie das von den PISA-Kriterien gefordert wird. Bei Hoffmanns „Sandmann“-Text bieten sich etwa die - immer aktueller werdenden - Fragen der geistigen Krankheit bzw. Normalität an. Der Protagonist leidet unter paranoiden Wahrnehmungsstörungen, die aber vom Autor nicht eindeutig als solche kenntlich gemacht werden, woraus die Spannung der Geschichte entsteht. Die Frage, ob seine Paranoia doch nicht vielleicht der (fiktionalen) Wirklichkeit entspricht, wird im Text nicht eindeutig beantwortet. Eine Aktivität, die das Reflektieren über den Inhalt der Geschichte in Gang setzen könnte, wäre etwa ein Rollenspiel, in dem ein 
Diskussionsleiter, ein Psychologe, ein Pädagoge (die „Störung“ ist als Kindheitstrauma entstanden), ein Freund des Protagonisten und die Verlobte des Protagonisten, die eine aufklärerisch herablassende Auffassung von geistigen „Defekten“ vertritt, in kleinen Runden ihre Meinungen formulieren. Die Argumente, die aus den Rollen heraus formuliert werden, müssen großteils aus dem Text geschöpft werden und tragen so zur Verbesserung des Leseverstehens bei, gleichzeitig betreffen sie aber die nichtfiktionale Lebenswelt der Studierenden. Eine „Humanisierung“ der Diskutierenden ist hier geradezu unumgänglich.

\section{Literaturgeschichte als Lesekompetenz: Verluste? Zusammenfassung}

Nun zusammenfassend die Negativa der vorgestellten Auffassung von Literaturunterricht und die Gründe, warum diese doch in Kauf zu nehmen sind. Zunächst stellt sich die Frage, worauf man verzichtet, indem man sich für kurze, erzählende Texte als die textuelle und methodische Basis von epochengeschichtlich angelegten literarischen Kursen entscheidet? Es bleiben vor allem die „kanonischen“ Texte außen vor, die meistens Romane oder längere Dramen sind. Einen Faust werden die angehenden DaF-LehrerInnen nicht „persönlich“ kennen lernen, einen Hans Biberkopf auch nicht; hier müssen die Vorlesungen Ersatz bieten. Es erscheint aber nicht ganz ausgeschlossen, dass die Studierenden diese Werke kursbegleitend oder nach dem Kurs doch selbst in die Hand nehmen werden, denn die Lesekompetenz - die Einsicht, Wesentliches in literarischen Texten suchen zu wollen und finden zu können -, die hierzu primär notwendig ist, haben sie vielleicht im Literaturunterricht erworben.

Worauf verzichtet man sonst noch, wenn man den organisatorischen Rahmen der Stunde größtenteils der „Diskussionsleitung “ von Studierenden überlässt? Auf die interpretatorische Vollständigkeit, soweit sie überhaupt erreichbar ist. Trotzdem finde ich es im Licht der vorangehenden Überlegungen sinnvoll, diesen textanalytischen Schwerpunkt in diesem Fall dem "diskussionstechnischen“ unterzuordnen. Es ist kaum ein kompetenter Leser zu denken, der ohne Gedankenaustausch über „fortlaufende“ Texte auskommt, und eine kompetente Lehrerpersönlichkeit wohl auch nicht.

\section{Literatur:}

http://pisa.ipn.uni-kiel.de/index.html

http://www.msmt.cz/pro-novinare/vysledky-mezinarodniho-vyzkumu-pisa-2006 